\title{
Atomistic description of the electronic structure of T-shaped quantum wires
}

\author{
J. Aizpurua ${ }^{\mathrm{a}, *}$, Garnett W. Bryant ${ }^{\mathrm{a}}$, W. Jaskólski ${ }^{\mathrm{b}}$ \\ ${ }^{a}$ National Institute of Standards and Technology, 100 Bureau Drive, Gaithersburg, MD 20899-8423, USA \\ ${ }^{\mathrm{b}}$ Instytut Fizyki, UMK, Grudziądzka 5, 87-100 Torún, Poland
}

\begin{abstract}
We use an atomistic tight-binding theory to study arrays of T-shaped GaAs/AlGaAs quantum wires. An atomistic approach allows us to provide a theory of the electron and hole states in $\mathrm{T}$ shaped wires without the approximations that limit effective mass models. We calculate the band structure of electron and holes in periodic T-wire arrays and in arm wells and compare with the results of effective mass theory. We determine the dispersion of single-particle states in a T-wire array and compare energies for arm-well and T-wire states.

Published by Elsevier Science Ltd.
\end{abstract}

Keywords: T-shaped wires; Excitons; Empirical tight-binding

\section{Introduction}

Semiconductor nanostructures are being intensively studied because they have electronic and optical properties very different from bulk properties. T-shaped wires, which can be built as an intersection of two quantum wells, are ideal candidates to study two-dimensional confinement of electrons, holes and excitons. In particular, enhanced excitonic binding has been reported in these wires $[1,2]$. Most recently, the coexistence of excitons with an electronhole plasma has been observed for high concentrations of charge carriers in T-shaped wires [3] and features of the photoluminescence (PL) spectra have been explained in terms of highly correlated many-body effects [4]. These results demand a renewed effort to develop a complete theory of electronic and excitonic states in T-shaped structures. Previous theoretical approaches for the $\mathrm{T}$ wire electronic structure have used an isotropic effective mass models better suited to describe the individual wells than the entire wire structure [5-7]. However, excitonic effects depend critically on which effective mass model is used. Here we present an empirical tight-binding (ETB) approach to describe the electronic structure of $\mathrm{T}$-shaped quantum wires.

\footnotetext{
* Corresponding author. Tel.: + 1-301-9758-560; fax: + 1-301-9901-350.

E-mail address: aizpurua@nist.gov (J. Aizpurua).
}

\section{Atomistic theory for periodic structures}

In the ETB method the Hamiltonian is represented in terms of an orthogonal basis set of atomic-like orbitals $\{|\alpha, J\rangle\}$, where $\alpha$ denotes a given orbital and $J$ an atomic site. In our model, each atom is described by orbitals $\left(\mathrm{s}, \mathrm{p}_{x}\right.$, $\mathrm{p}_{y}, \mathrm{p}_{z}$, and $\mathrm{s}^{*}$ ), and their interaction is restricted to on-site and nearest neighbors. An fcc lattice with a two atoms basis describes the zincblende structure of GaAs and AlAs. The tight-binding parameters

$t_{\alpha^{\prime} \alpha, \mathbf{R}_{J^{\prime}} \mathbf{R}_{J}}=\left\langle\alpha^{\prime}, J^{\prime}|H| \alpha, J\right\rangle$,

are taken from Vogl et al. [8]. In this model, spin-orbit effects are ignored. However, they can easily be included in our tight-binding theory. We do not include lattice relaxation in the present study since AlAs and GaAs have similar lattice parameters and there is almost no lattice mismatch.

The tight-binding wave function $\Psi(\mathbf{r})$ is expressed as a linear combination of the atomic orbitals $\phi_{\alpha}\left(\mathbf{r}-\mathbf{R}_{\mathbf{J}}\right)$ $=\langle\mathbf{r} \mid \alpha, J\rangle$ :

$\Psi(\mathbf{r})=\sum_{J} \sum_{\alpha} c_{\alpha J} \phi_{\alpha}\left(\mathbf{r}-\mathbf{R}_{\mathbf{J}}\right)$.

For periodic arrays of wires, we work in the reciprocal space wire with the use of Bloch waves

$\Psi_{\mathbf{k}}(\mathbf{r})=\frac{1}{\sqrt{N}} \sum_{\mathbf{S}} \sum_{\alpha, J} c_{\alpha, J, \mathbf{k}} \mathrm{e}^{\mathrm{ik} \cdot \mathbf{S}} \phi_{\alpha}\left(\mathbf{r}-\mathbf{R}_{\mathbf{J}}-\mathbf{S}\right)$ 


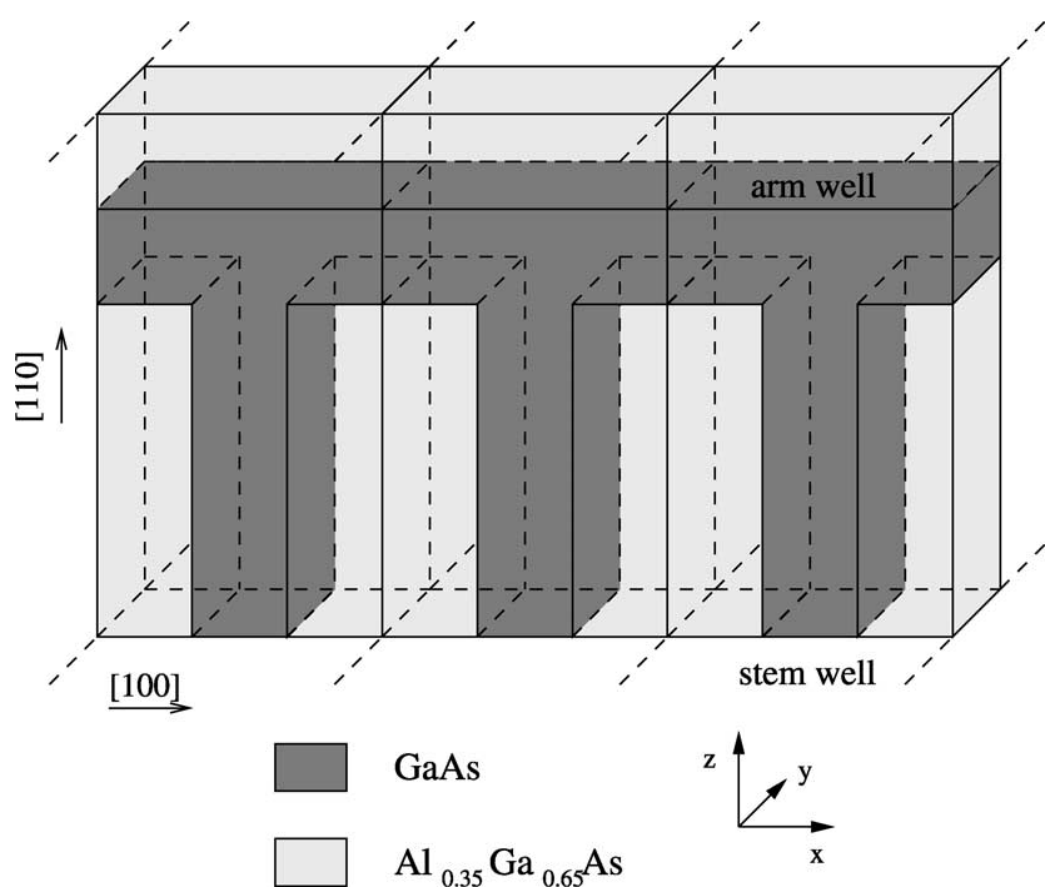

Fig. 1. Scheme of a GaAs/AlGaAs T-shaped wire array. The wires extend along the $Y$ axis, perpendicular to the arm well which also extends along the $X$ direction. We consider T-wires made from [110] arm wells as in the experimental structures.

The sum over $J$ includes all atoms in the GaAs T-wire and in the AlGaAs surrounding box that defines the super cell. The alloy composition in the barrier is modeled by using a composition-weighted energy of the tight binding parameters. Here we consider $\mathrm{Al}_{0.35} \mathrm{Ga}_{0.65} \mathrm{As}$. A summation over the super cells in the periodic array is described by the lattice vector $\mathbf{S}$ defined along $X$ by the wire periodicity $(\approx 13.5 \mathrm{~nm})$ and along $Y$ by the atomic periodicity $(0.396 \mathrm{~nm})$, as shown in Fig. 1 . The electronic structure is obtained by diagonalizing the tight-binding Hamiltonian

$H_{\alpha^{\prime}, J^{\prime}, \alpha, J}(\mathbf{k})=\left\langle\alpha^{\prime}, J^{\prime}, \mathbf{k}|H| \alpha, J, \mathbf{k}\right\rangle=\sum_{\mathbf{S}} \mathrm{e}^{\mathrm{i} \mathbf{k} \cdot \mathbf{S}} t_{\alpha^{\prime} \alpha, \mathbf{R}_{\mathbf{J}^{\prime}}+\mathbf{S} \mathbf{R}_{\mathbf{J}}} \cdot$

The inter-atomic orbital interaction is limited to nearest neighbors. Effectively the atoms interact with nearest neighbors at the same super cell or with atoms that become nearest neighbors in an adjacent super cell after a phase shift.

\section{Results}

The T-wire PL is red-shifted from the PL of the isolated wells $[1,2]$. This shift is connected with the trapping of the electrons and holes in the T-wire and with an enhancement of the binding energy of the exciton in the quasi 1D wire. We calculate the energies for holes and electrons in an array of T-wires by using the ETB. We compare these energies with the energies of an arm well with the same thickness. The single-particle binding energy for electrons and holes, i.e. the difference between the energy in the T-wire and in the arm well is shown in Fig. 2 for different thicknesses of the arm well forming the T-wire. The thickness of the [100] stem well is taken as close as possible to the thickness of the [110] arm well. These two thicknesses need not be exactly the same because the [100] and [110] layer spacing are different. The wire spacing is taken at least three times larger than the stem well thickness. This value ensures little effect on the energy of the wire states as a result of the interwire coupling.

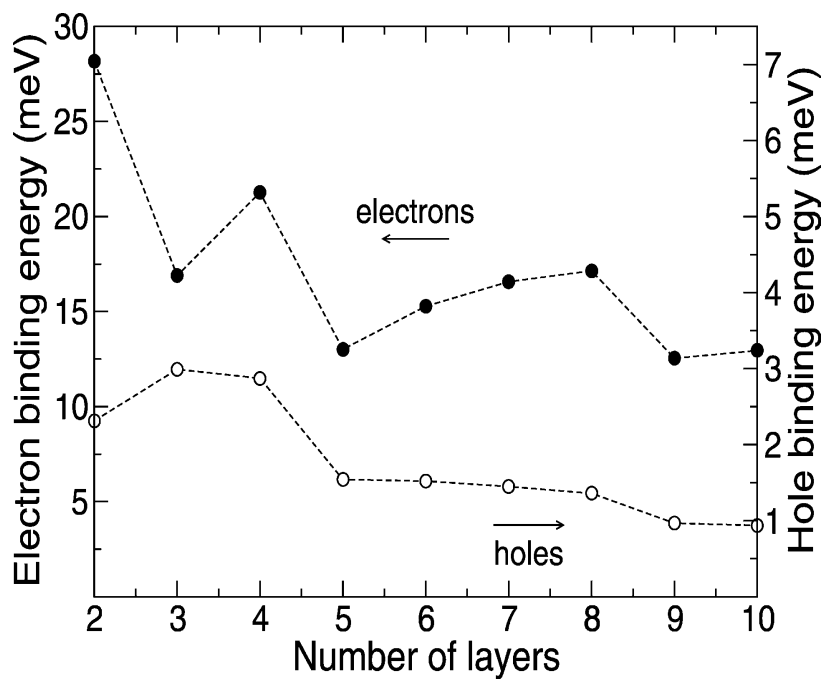

Fig. 2. Single-particle electron (black circles) and hole (white circles) binding energy in a T-wire as a function of the number of layers in the [110] direction. 

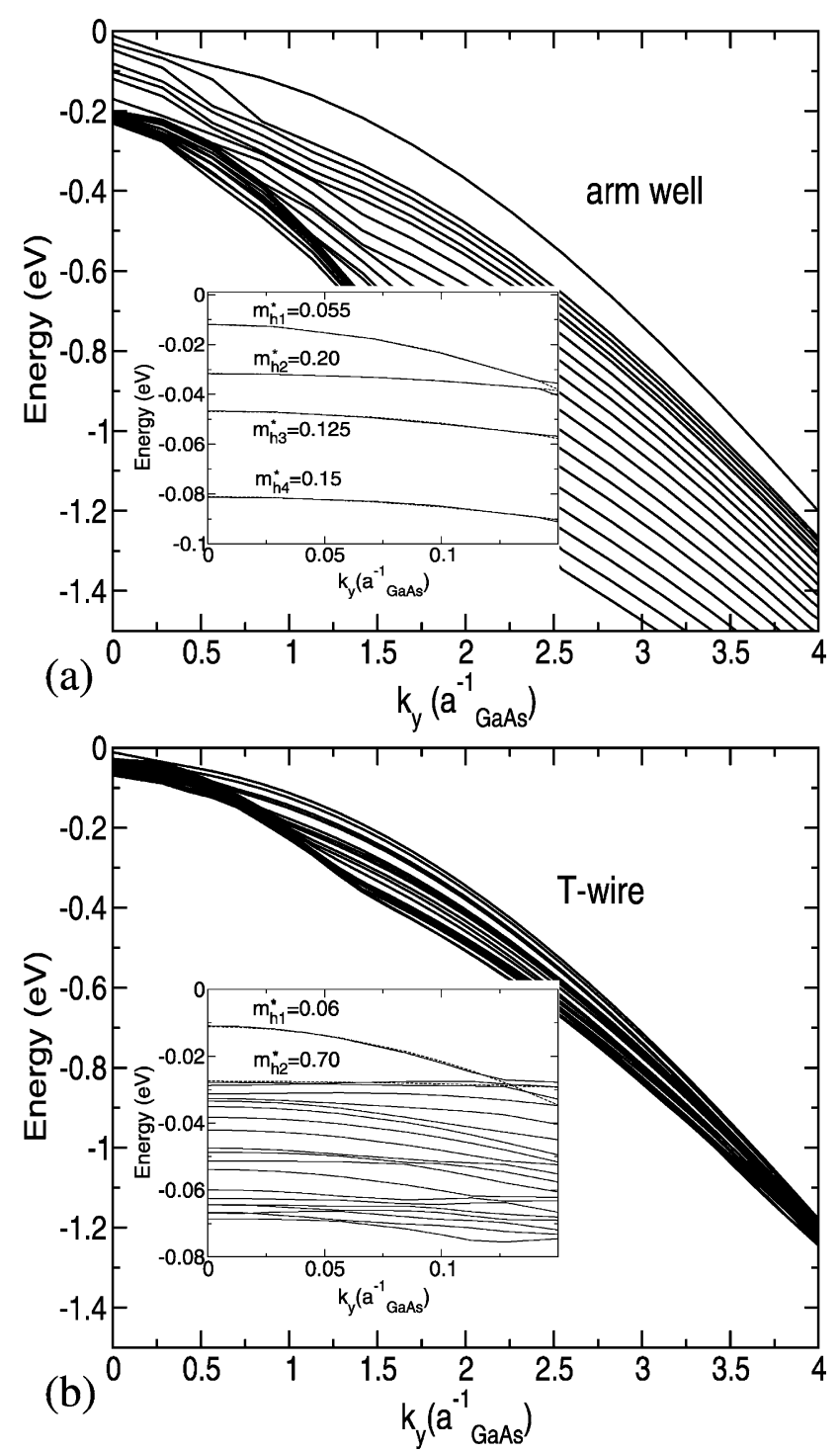

Fig. 3. Hole state dispersion along $Y$ for an arm well (a) and a T-wire (b). The thickness of arm well is $4 \mathrm{~nm}$ along the [110] direction. Insets show the Brillouin zone center enhanced. The effective masses are in units of the free electron mass.

Holes have a binding energy which is always smaller than $2.5 \mathrm{meV}$ even for very thin wires. For thicker wires the binding energy is below $1 \mathrm{eV}$. On the other hand, electrons have a larger binding energy which can reach $30 \mathrm{meV}$ for very thin wells. The fluctuations of the binding energy are connected with differences in the thickness of the stem well with respect to the arm well which have an effect in raising or lowering the binding energy in as much as $\approx 5 \mathrm{meV}$. Within this uncertainty due to the stem well thickness the curves follow a monotonic behavior. As a consequence of the strong electron trapping and the weaker hole trapping, the electron is more localized to the T-wire than the hole. This disparity in localization directly affects exciton formation [5-7] and should be crucial in the formation of electron-hole plasmas in highly populated T-wires where many-body effects are claimed to play the main role [4].

We determine now the dispersion of holes along the wire axis. In Fig. 3 we show the dispersion along $Y$ of the lowest hole states for the arm well (a) and wire (b) where the thickness of the arm well and stem well are 4 and $3.95 \mathrm{~nm}$, respectively. In effective mass theory, the lowest well hole-state is a doubly degenerate heavy hole. Tightbinding theory shows that this degeneracy is lifted because symmetry is broken by the atomic structure of the well/barrier interface. Effective masses are different for states split by the interface symmetry breaking [see the inset in Fig. 3(a)]. The effective mass of the second lowest hole state $m_{\mathrm{h} 2}^{*}$ along the $Y$ axis is four times larger than the lowest hole state $m_{\mathrm{h} 1}^{*}$. There is also a difference in the dispersion and therefore in the effective masses of holes in [110] and [100] directions with a change of the light/ heavy character.

The dispersion of holes in a T-wire shows features similar to those of the isolated arm well. There is a degeneracy break due to the well/barrier interface and the ground state corresponds to a light hole along the wire axis with mass $m_{\mathrm{h} 1}^{*}=0.06$. The second state is a heavy hole-like state in this direction with effective mass $m_{\mathrm{h} 2}^{*}=0.70$.

The values for the effective masses along the wire direction are similar to those used in effective mass theory, although the effective mass of the second hole state in a T-wire is heavier. The atomistic approach also shows an asymmetry in the $X$ and $Y$ directions that is not considered in previous models and will be reported in future work. This asymmetry causes a reversal of the heavy/light hole behavior in the [100]/[110] directions.

\section{Conclusion}

An atomistic ETB description of electrons and holes in T-shaped wires is used to determine single carrier binding energy and dispersion. Electrons are more confined to the wire than holes. The properties of excitons and electronhole plasmas in such structures will be governed by those differences in trapping.

\section{References}

[1] T. Someya, H. Akiyama, H. Sakaki, Enhanced binding energy of onedimensional excitons in quantum wires, Phys. Rev. Lett. 76 (1996) 2965-2968.

[2] J. Hasen, L.N. Pfeiffer, A. Pinczuk, H.U. Baranger, K.N. West, B.S. Dennis, Large excitonic confinement in asymmetric quantum $\mathrm{T}$ wires, Superlattices Microstruct. 22 (1997) 359-364.

[3] J. Rubio, L. Pfeiffer, M.H. Szymanska, A. Pinczuk, S. He, H.U. Baranger, P.B. Littlewood, K.W. West, B.S. Dennis, Coexistence of excitonic lasing with electron-hole plasma spontaneous emission in 
one-dimensional semiconductor structures, Solid State Commun. 120 (2001) 423-427.

[4] H. Akiyama, L.N. Pfeiffer, A. Pinczuk, K.W. West, M. Yoshita, Observation of large many-body Coulomb interaction effects in a doped quantum wire, Solid State Commun. 122 (2002) 169-173.

[5] G.W. Bryant, P.S. Julienne, Y.B. Band, Exciton trapping, binding and diamagnetic shift in T-shaped quantum wires, Superlattices Microstruct. 20 (1996) 601-607.
[6] M.H. Szymanska, P.B. Littlewood, R.J. Needs, Excitons in T-shaped quantum wires, Phys. Rev. B 63 (2001) 205317.

[7] G.W. Bryant, Y.B. Band, T-shaped quantum wires in magnetic fields: Weakly confined magneto excitons beyond the diamagnetic limit, Phys. Rev. B 63 (2001) 115304.

[8] P. Vogl, H.P. Hjalmarson, J.D. Dow, A semi-empirical tight-binding theory of the electronic structure of semiconductors, J. Phys. Chem. Solids 44 (1983) 365-378. 